\title{
Efecto del vanadio en la forja en tibio de un acero con contenido medio de carbono
}

\author{
C. $\operatorname{García-Mateo~}^{(*)}$ J.L. Romero $^{(*)}$ y J.M. Rodríguez-Ibabe ${ }^{(*)}$
}

\begin{abstract}
Resumen En el presente trabajo se analiza la influencia del vanadio en la microestructura y en la resistencia mecánica de un acero con contenido medio de carbono, tras ser deformado por compresión plana a temperaturas inferiores a $900^{\circ} \mathrm{C}$. Los carbonitruros de vanadio no disueltos afinan la microestructura y junto a los reprecipitados durante el enfriamiento contribuyen al endurecimiento por precipitación, manteniendo la resistencia mecánica y logrando una tenacidad aceptable al bajar la temperatura de deformación.
\end{abstract}

Palabras clave: Aceros microaleados. Forja en tibio. Propiedades mecánicas. Tenacidad.

\section{Effect of vanadium in the warm forging of a medium carbon steel}

\begin{abstract}
In the present work the influence of vanadium on the microstructure and mechanical properties of a medium carbon steel forged below $900{ }^{\circ} \mathrm{C}$ is analysed. Undisolved $\mathrm{V}(\mathrm{CN})$ refines the microstructure and together with reprecipitated particles during cooling contribute to strengthening. Both effects maintain mechanical strength levels without decreasing toughness values.
\end{abstract}

Keywords: Microalloyed steels. Warm forging. Mechanical properties. Toughness.

\section{INTRODUCCIÓN}

Se entiende por forja en tibio de aceros la deformación a temperaturas en el rango $500-900^{\circ} \mathrm{C}$. Esta ruta de fabricación ofrece ventajas sobre las convencionales de forja en caliente o en frío. Con respecto a la primera, se consigue una mejora de las dimensiones finales y mejor acabado superficial, respecto a la segunda se requiere la aplicación de cargas menores (1-5). La introducción del vanadio en aceros que van a ser conformados en tibio tiene distintos objetivos. Por una parte, se trata de aprovechar las partículas no disueltas de carbonitruros de vanadio para anclar las juntas de grano austeníticas, obteniendo así un tamaño de grano fino que redunde en una buena resistencia mecánica y una buena tenacidad. Por otra parte, el vanadio disuelto permite obtener un rendimiento de este elemento para conseguir endurecimiento por precipitación (6). El objetivo de este estudio va a ser el poder

(*) Centro de Estudios e Investigaciones Técnicas de Guipúzcoa (CEIT) y ESII de San Sebastián. Po Manuel de Lardizabal 15. 20009-San Sebastián (España). emplear, gracias a los anteriores efectos del vanadio, aceros con menor contenido de carbono para unos valores dados de resistencia mecánica, lo que incide directamente en una mejora de los valores de tenacidad.

\section{MATERIAL Y PROCEDIMIENTO EXPERIMENTAL}

Se ha estudiado un acero microaleado al vanadio, con $0,24 \% \mathrm{C}, 1,56 \% \mathrm{Mn}, 0,28 \% \mathrm{Si}, 0,18 \%$ $\mathrm{V}$ y $0,0099 \% \mathrm{~N}$, suministrado en forma de palanquilla de $50 \mathrm{~mm}$ de espesor. Se realizaron ensayos de dilatometría en el CENIM para determinar las temperaturas de transformación $A c_{1}$ y $A c_{3}$. Para simular el proceso de forja, se han realizado ensayos de compresión plana sobre muestras de $10 \times 25$ $\times 50 \mathrm{~mm}$ mecanizadas desde la palanquilla. Las muestras se calentaron a las temperaturas de ensayo $\left(870,835,800\right.$ y $\left.720^{\circ} \mathrm{C}\right)$ durante $10 \mathrm{~min}$, para posteriormente serles aplicada una deformación $\varepsilon=0,3$ a una velocidad de deformación constante de $10 \mathrm{~s}^{-1}$. A continuación, algunas muestras se dejaron enfriar 
al aire y otras con aire comprimido $\left(\sim 4^{\circ} \mathrm{C} / \mathrm{s}\right)$. Las probetas ensayadas se cortaron longitudinalmente para su estudio mediante microscopía óptica y electrónica, tras ser pulidas y posteriormente atacadas con Nital $2 \%$. Para la caracterización de las propiedades mecánicas, se extrajeron de las muestras deformadas en compresión plana, probetas de tracción y Charpy con entalla en V de $5 \times 10 \mathrm{~mm}$. Así mismo, se extrajeron réplicas de carbono para analizar los precipitados de vanadio en un microscopio electrónico de transmisión (TEM).

\section{RESULTADOS}

Con los ensayos dilatométricos, se han determinado las temperaturas de comienzo y final de transformación $A c_{1}=725^{\circ} \mathrm{C} \mathrm{y} A c_{3}=810^{\circ} \mathrm{C}$, pudiéndose fijar las temperaturas de los ensayos en las regiones $\alpha, \alpha+\gamma$ y $\gamma$. Por otro lado, el tamaño de grano austenítico medio, medido a $870{ }^{\circ} \mathrm{C}$, ha sido de $5 \mu \mathrm{m}$, encontrándose un $94 \%$ de los granos por debajo de las $9 \mu \mathrm{m}$.

Las estructuras obtenidas en las muestras con enfriamiento lento son principalmente ferrítico-perlíticas con islotes bainíticos y tamaños de grano medios muy finos $(<4,5 \mu \mathrm{m}$ en todos los casos). Los enfriamientos acelerados varían los productos de la transformación y la fracción volumétrica de bainita aumenta en detrimento de las fracciones volumétricas de ferrita y perlita. La tabla I resume los parámetros microestructurales cuantificados. En la figura 1 , se observan distintas micrografías típicas de las muestras deformadas a 870 y $720^{\circ} \mathrm{C}$, con enfriamientos lento y rápido, pudiendo observarse en todas ellas lo fino de la microestructura, si bien destaca que las muestras deformadas a $720{ }^{\circ} \mathrm{C}$ pre- sentan un predominio de los granos de ferrita alargados en el plano normal al de deformación.

Los valores medios obtenidos en los ensayos de tracción se resumen en la tabla I. En todas las muestras estudiadas, el límite elástico se encuentra en el rango de 460-640 MPa, correspondiendo el valor más alto a la muestra conformada a $720{ }^{\circ} \mathrm{C}$ con enfriamiento al aire. La resistencia máxima a tracción se encuentra entre los valores 760-960 $\mathrm{MPa}$, pudiéndose apreciar una gran diferencia entre los valores obtenidos en los enfriamientos acelerados y los realizados al aire. Estos últimos tienen valores en torno a 760-800 $\mathrm{MPa}$, mientras que los enfriados aceleradamente superan los $960 \mathrm{MPa}$. Los valores de reducción de área obtenidos son, asimismo, elevados, superiores al 50, e incluso al $60 \%$, en las muestras con enfriamiento lento alcanzando, sin embargo, entre el 42 y el $48 \%$ en las muestras con enfriamiento acelerado.

Los resultados de los ensayos Charpy a temperatura ambiente y a $0{ }^{\circ} \mathrm{C}$, se han normalizado a probeta estándar de $10 \times 10 \mathrm{~mm}$ y se resumen en la tabla II. Tal y como la reducción de área indicaba, la menor energía absorbida corresponde a las muestras deformadas a $870{ }^{\circ} \mathrm{C}$, que, además, son las únicas en presentar un comportamiento frágil a ambas temperaturas de ensayo. El resto de las muestras ensayadas han revelado un comportamiento dúctil a ambas temperaturas, siendo, además, las energías absorbidas muy similares en ambos casos. En la figura 2, se observan fractografías correspondientes a muestras de 870 y $835^{\circ} \mathrm{C}$, en las que se aprecia el distinto tipo de fractura que se ha producido entre ambas.

La extracción de réplicas de carbono y su posterior análisis en el microscopio electrónico de transmisión han proporcionado información sobre el

TABLA I.- Parámetros microestructurales y propiedades mecánicas

TABLE I.-Microstructural parameters and mechanical properties

\begin{tabular}{|c|c|c|c|c|c|c|}
\hline Temp. $\left({ }^{\circ} \mathrm{C}\right)$ & $\mathrm{V}_{\alpha}(\%)$ & $\mathrm{D}_{\alpha}(\mu \mathrm{m})$ & Bainita $(\%)$ & $\begin{array}{c}\sigma_{0.2 \%} \\
(\mathrm{MPa})\end{array}$ & $\begin{array}{c}\text { U.T.S. } \\
(\mathrm{MPa})\end{array}$ & $\begin{array}{c}\text { R.A. } \\
(\%)\end{array}$ \\
\hline 870 & $45,4 \pm 3$ & $4,5 \pm 0,2$ & $\cong 21$ & 478,5 & 804,2 & 54 \\
835 & $\mathrm{nc}$ & $\mathrm{nc}$ & $\mathrm{nc}$ & 529,5 & 789,0 & 67 \\
800 & $41 \pm 2$ & $1,3 \pm 0,1$ & $\cong 22$ & 520,5 & 765,2 & 67 \\
720 & $54 \pm 2$ & $3,6 \pm 0,2$ & $<7$ & 637,5 & 765,3 & 67 \\
$870^{*}$ & $39 \pm 2$ & $1,7 \pm 0,1$ & $21 \pm 2$ & 541,0 & 975,4 & 42 \\
$800^{*}$ & $51 \pm 3$ & $2,3 \pm 0,1$ & $47, \pm 3$ & 457,2 & 960,9 & 48 \\
$720 *$ & $49 \pm 3$ & $4,1 \pm 0,2$ & $\cong 14$ & 552,1 & 983,8 & 46 \\
\hline
\end{tabular}

*Muestras sometidas a enfriamiento acelerado. nc: no cuantificado. 

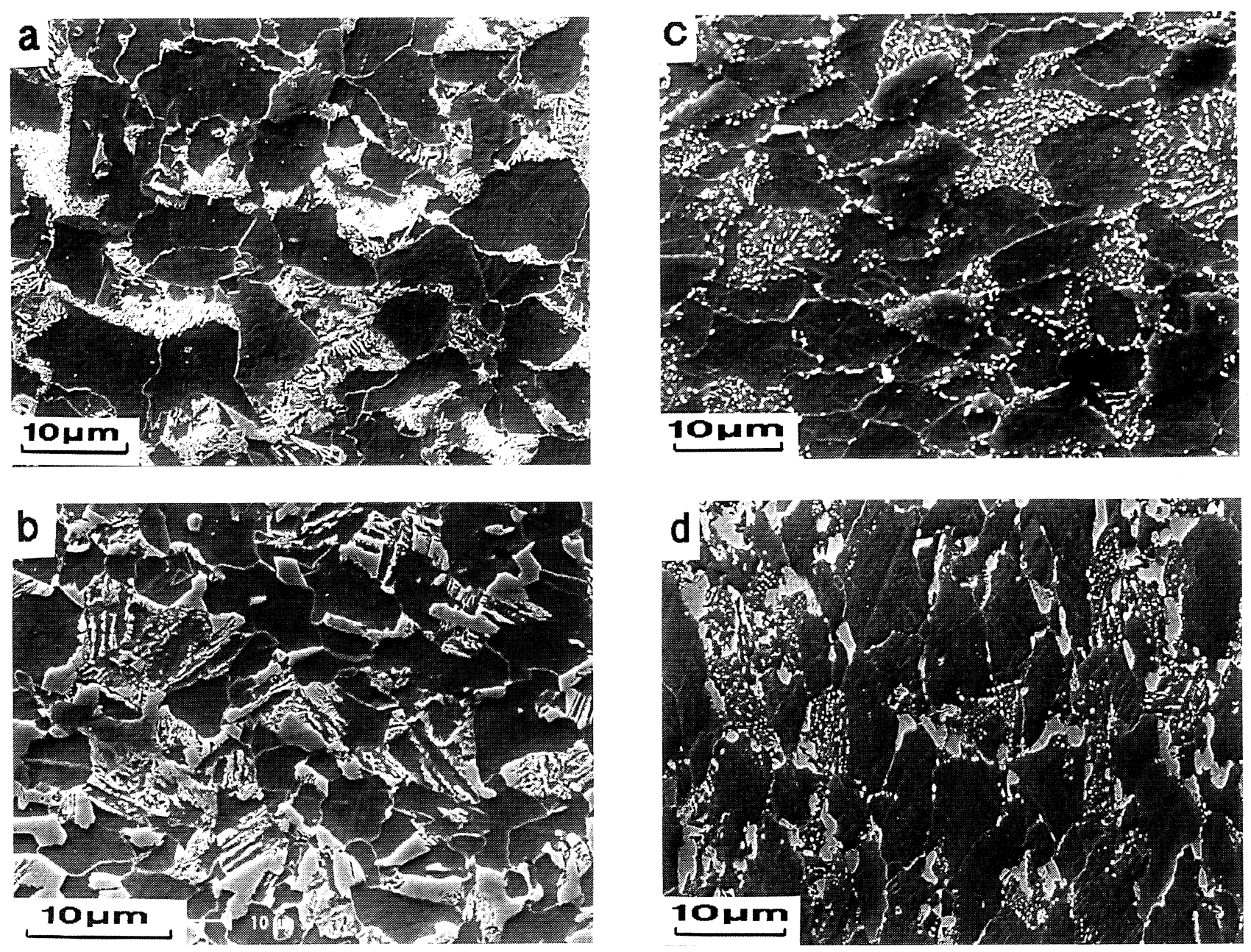

FIG. 1.- Microestructuras de muestras deformadas a a) $870{ }^{\circ} \mathrm{C}$ (enf. lento), b) $870{ }^{\circ} \mathrm{C}$ (enf. rápido), c) $720{ }^{\circ} \mathrm{C}$ (enf. lento) y $d$ ) $720^{\circ} \mathrm{C}$ (enf. rápido)

FIG. 1.- Microstructures of deformed specimens at a) $870^{\circ} \mathrm{C}$ (air cooled), b) $870^{\circ} \mathrm{C}$ (accelerated cooled), c) $720^{\circ} \mathrm{C}$ (air cooled) and d) $720^{\circ} \mathrm{C}$ (accelerated cooled)

TABLA II.- Resultados del ensayo Charpy

TABLE II.- Results of Charpy test

\begin{tabular}{|c|c|c|}
\hline Temp, ${ }^{\circ} \mathrm{C}$ & $\mathrm{CVN} \mathrm{T}_{\mathrm{R}}, \mathrm{J}$ & $\mathrm{CVN} \mathrm{0}{ }^{\circ} \mathrm{C}, \mathrm{J}$ \\
\hline 870 & 46,0 & 35,5 \\
835 & 92,1 & 95,5 \\
800 & 114,2 & 112,2 \\
720 & 107,3 & 94,6 \\
\hline
\end{tabular}

Valores medios de resiliencia obtenidos tras normalizar a probeta estándar de $10 \times 0 \mathrm{~mm}$.

tamaño medio de los $\mathrm{V}(\mathrm{CN})$. En la figura 3, se muestra la distribución de diámetros de partículas a 870 y $800{ }^{\circ} \mathrm{C}$ (enfriados al aire) y la del material de partida, habiéndose obtenido la distribución sobre una población de 900 partículas en cada caso. Se observa que, conforme aumenta la temperatura de calentamiento y deformación, las partículas van teniendo un mayor tamaño, a pesar de lo cual la mayor parte de éstas se mantiene por debajo de los $30 \mathrm{~nm}$.

\section{DISCUSIÓN}

$\mathrm{Al}$ calentar las muestras hasta una temperatura máxima de $870{ }^{\circ} \mathrm{C}$, durante un intervalo de tiempo breve, sólo una pequeña parte del vanadio que la red austenítica podría mantener disuelto en condiciones de equilibrio $(\approx 60 \%$ del $\mathrm{V})$ se encuentra en ese estado (7). Esto permite mantener una distribución fina de partículas que actúan como puntos de anclaje de las juntas austeníticas, cuyo tamaño medio es de $5 \mu \mathrm{m}$ y, además, mantienen una parte importante de su efecto endurecedor por precipitación (6-8 y 9). Tal y como se muestra en la figura 3 , la distribución de tamaños de las partículas se va desplazando hacia la derecha (respecto a la del 

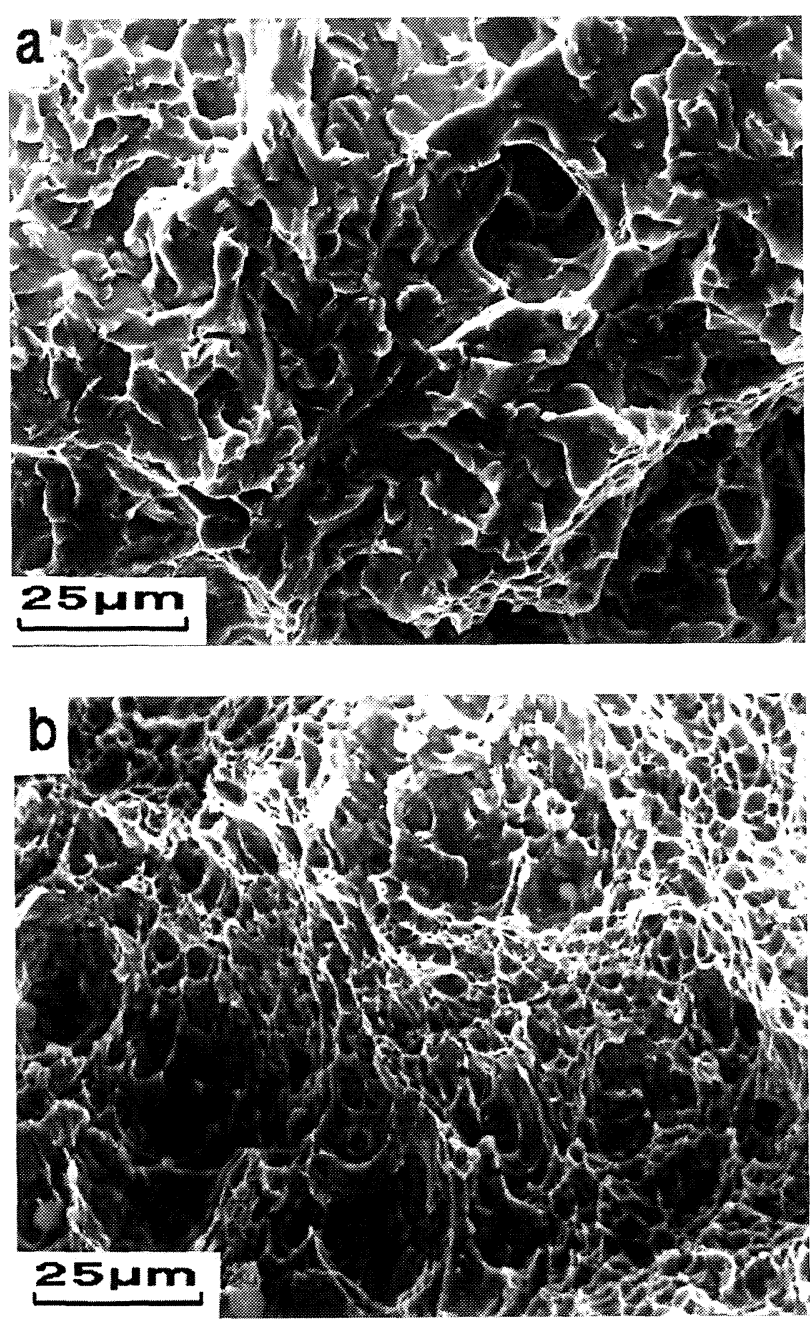

FIG. 2.- Fractografías correspondientes a muestras enfriadas al aire tras deformación a a) $870{ }^{\circ} \mathrm{C}$ y b) $835^{\circ} \mathrm{C}$.

FIG. 2.- Fractographies of air cooled specimens after forming at a) $8700^{\circ} \mathrm{C}$ and b) $835^{\circ} \mathrm{C}$.

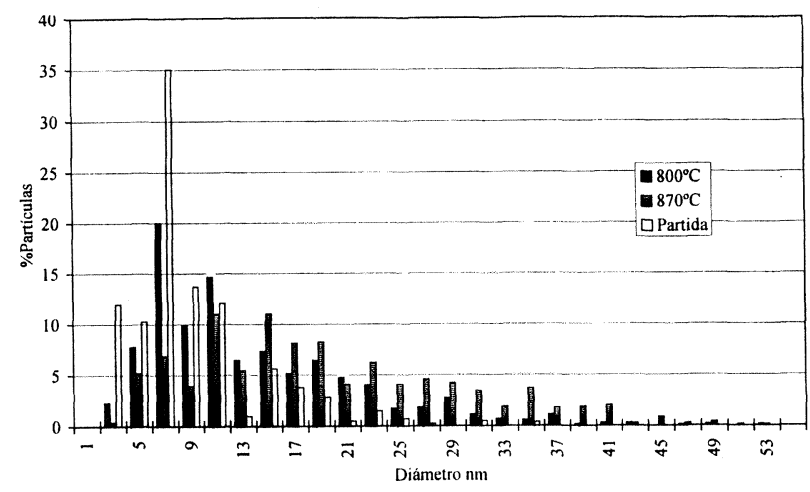

Fig. 3. - Histogramas de los diámetros medios de los carbonitruros de vanadio

FIG. 3.- Histograms of the mean diameters of $V(C N)$.

material de partida) conforme aumenta la temperatura de calentamiento, siendo más fina a 800 que a
$870{ }^{\circ} \mathrm{C}$. El aumento del tamaño de las partículas a estas temperaturas se debe al engrosamiento de las mismas por efecto de la temperatura (6).

Es importante resaltar la influencia que la presencia de bainita en distintas proporciones, según tratamiento, ejerce sobre el comportamiento mecánico de la microestructura. Como se observa en la tabla I, en los enfriamientos acelerados disminuye el límite elástico, al tiempo que la UTS aumenta, siendo éste un comportamiento típico de estructuras bainíticas (10). Pero a $870{ }^{\circ} \mathrm{C}$, se observa, por contra, un aumento del límite elástico respecto a su homólogo de enfriamiento lento, lo que puede considerarse consecuencia de una más fina reprecipitación de vanadio en la ferrita a causa del enfriamiento acelerado, así como al afino del tamaño de grano ferrítico logrado, más importante a esta temperatura que en el resto de los casos.

El conformado del material a $720^{\circ} \mathrm{C}$, prácticamente en la región ferrítica, incide en las propiedades mecánicas al producirse un endurecimiento por deformación, así como el endurecimiento por precipitación, ya que la distribución de partículas será en la práctica muy similar a la del material de partida. Aun así, el pequeño porcentaje transformado a austenita hace que en el enfriamiento rápido aparezca más bainita que en el lento, produciéndose una disminución en el límite elástico.

Los ensayos de tenacidad Charpy muestran que, a pesar de tener buenos valores de límite elástico y de UTS, la tenacidad del material se mantiene en niveles también aceptables. Esto concuerda con datos publicados por Naylor (11) para aceros con contenidos medios de carbono y microaleados con vanadio, en que logra resiliencias superiores a los $100 \mathrm{~J}$ mediante deformación en tibio. Sólo a 870 ${ }^{\circ} \mathrm{C}$, el material presenta un comportamiento frágil, tanto para $0{ }^{\circ} \mathrm{C}$ como para temperatura ambiente, lo que puede deberse a la heterogeneidad del tamaño de grano austenítico, que, en algunos casos, alcanza las $15 \mu \mathrm{m}$ y que queda corroborada por la existencia de facetas $(>15 \mu \mathrm{m})$ en la superficie de fractura.

\section{CONCLUSIONES}

- Se ha examinado la relación entre la microestructura y las propiedades mecánicas de un acero con contenido medio de carbono y microaleado con vanadio, en el rango de temperaturas entre $700-900{ }^{\circ} \mathrm{C}$.

- Una distribución de partículas finas en la austenita impide su crecimiento, obteniendo a $870{ }^{\circ} \mathrm{C}$ un tamaño medio de $5 \mu \mathrm{m}$, transformando a una microestructura final fina.

- El alto contenido de manganeso eleva la templabilidad del acero, proporcionando estructuras 
mixtas (ferrita+perlita+bainita) incluso en los enfriamientos al aire.

- Un límite elástico más elevado y una menor resistencia a tracción son las diferencias fundamentales entre los enfriamientos lentos y los rápidos, siendo esto consecuencia de la mayor presencia bainítica en estos últimos.

- En el rango de temperaturas ensayado, la cantidad de vanadio disuelto es pequeña, lo que hace de vital importancia que el material suministrado posea una distribución fina de partículas. Esto afecta a distintos mecanismos de endurecimiento, al impedir así el crecimiento del grano austenítico, lo que conduce a estructuras más finas tras la transformación, y por otro lado, a producir endurecimiento por precipitación si las partículas no engrosan de forma importante.

\section{Agradecimiento}

Los autores desean agradecer al Gobierno Vasco el soporte financiero para realizar el proyecto en el que se engloba este trabajo y a C. García de Andrés (CENIM) su ayuda al realizar los ensayos de dilatometría. Asimismo, C. García Mateo agradece a la Asociación de Amigos de la Universidad de Navarra la concesión de una beca para investigación.

\section{REFERENCIAS}

(1) HuSAIN, Z. ECSC Information Day. Engineering Steel (12 Oct. 1995) VDEh, Dusseldorf (Alemania).

(2) Pollard, R.W. y Stainnaker, L.L. Tool Prod.., 47 (7), 1981: 88-89.

(3) Suzuki, T. Toyota Motors. Int. J. Match. Tools Manuf., 29 (1), 1989: 5-27.

(4) Aloi, N.E., Krauss, G., Matlock, D.K., Van Tyne, C.J. y ChenG, Y.W. 36th MWSP Conf. Proc., ISS-AIME, Vol. XXXII, 1995: 201-213.

(5) Reynolds, J.H. y NAYloR, D.J. Mater. Sci. Tech., 4 Jul. 1988: 586-602.

(6) Gladman, T. The Physical Metallurgy of Microalloyed Steels. $1^{a}$ Ed. The Institute of Materials, 1997.

(7) Rivas, A.L., Michal, G.M., Burnett, M.E. y MusolfF, C.F. Fundamentals and Applications of Microalloying Forging Steels. The Minerals, Metals and Materials Society, 1996: 159-172.

(8) MORRISON, W.B. Ironmaking Steelmaking, 22 (6), 1995 453-458.

(9) NAYLOR, D.J. Ironmaking Steelmaking, 16 (4), 1989: 246252.

(10) Mekkawy, M.F., El-Fawakhry, K.A., Mishrehy, M.L. y EISSA, M.M. Scand. J. Metall., 19, 1990: 246-256.

(11) NAYLOR, D.J. Ironmaking Steelmaking, 17 (1), 1990: 17-25. 